\title{
Changes in Rapid Eye Movement Sleep Associated with Placebo-Induced Expectations and Analgesia
}

\author{
Danièle Laverdure-Dupont, ${ }^{1,3}$ Pierre Rainville, ${ }^{2}$ Jacques Montplaisir, ${ }^{3}$ and Gilles Lavigne ${ }^{2,3}$ \\ ${ }^{1}$ Department of Physiology, Faculty of Medicine and 2Departments of Stomatology and Oral Health, Faculty of Dental Medicine, University of Montréal, \\ Montréal, Québec H3T 1J4, Canada, and ${ }^{3}$ Centre d'étude du Sommeil et des Rythmes Biologiques, Hôpital du Sacré-Cœur, Montréal H4J 1C5, Canada
}

The experience of a sensory event is extensively shaped by past experience and expectations. Placebo analgesia, one of the most studied models of expectation-mediated effects, can be induced by suggestion of analgesia and conditioning. The present study examined the possibility that sleep might contribute to the consolidation of new expectations and consequently influence the generation of expectationmediated placebo effects. Strong expectations of analgesia were generated before sleep by conditioning manipulations wherein the intensity of thermal pain stimulation was surreptitiously reduced after the application of a topical placebo cream. Expectations and placebo analgesic effects were measured the following morning and compared with those of a control daytime group without sleep. Although placebo effects were observed in both groups, correlation analysis suggests that the mediating effect of expectations on placebo responses was strongest in the overnight group. Moreover, after exposure to a convincing analgesia experience, the relative duration of rapid eye movement (REM) sleep decreased in subjects showing higher analgesic expectations and placebo responses the next morning. In a third group exposed to less consistent analgesic experiences before sleep, expectations reported in the morning were comparable with other groups. However, expectations were positively correlated with REM sleep and did not emerge as a significant mediator of the analgesic effect. Together, these findings show that sleep-related processes may influence the association between expectations and placebo analgesia and that REM sleep can predict placebo-induced expectations of pain relief. However, equivocal previous experience with treatments may significantly alter the relationship between relief expectation, REM sleep, and placebo effects.

\section{Introduction}

Expectations originate from the integration of current information with past experience and personal beliefs and can greatly shape the experience of a particular sensory event (Ploghaus et al., 2003; Koyama et al., 2005). Many pain studies have highlighted the pivotal role of expectation in the establishment of placeboinduced analgesic effects (Montgomery and Kirsch, 1997; Price et al., 1999; Benedetti et al., 2003). A combination of verbal suggestion and sensory conditioning is commonly used to induce placebo analgesia and is one of the most studied models of expectation-mediated effects (Price et al., 1999; Petrovic et al., 2002; Wager et al., 2004, 2007; Bingel et al., 2006). Because previous exposure to an effective treatment can enhance analgesic responses when compared with suggestion alone (Colloca and Benedetti, 2006; Colloca et al., 2008), placebo effects may result from learning processes involving the consolidation of expectationrelated effects. In recent years, the role of sleep in memory reprocessing and learning has been increasingly recognized (Walker and Stickgold, 2004), but its involvement in the consolidation of newly acquired expectations remains unexplored.

\footnotetext{
Received March 9, 2009; revised July 9, 2009; accepted Aug. 7, 2009

We thank the technicians at the Centre for the Study of Sleep and Biological Rhythms at Sacré-Coeur Hospital (Montréal, QC, Canada), particularly Maryse Parenteau and Hajar El Alaoui.

Correspondence should be addressed to Danièle Laverdure-Dupont, Centre d'étude du Sommeil et des Rythmes Biologiques, Hôpital du Sacré-Cœur de Montréal, 5400 boulevard Gouin Ouest, Montréal, QC H4J 1C5, Canada. E-mail: daniele.laverdure-dupont@umontreal.ca.

DOI:10.1523/JNEUROSCI.1224-09.2009

Copyright $\odot 2009$ Society for Neuroscience $\quad 0270-6474 / 09 / 2911745-08 \$ 15.00 / 0$
}

Training and sleep deprivation studies have highlighted the differential contribution of separate sleep stages to the acquisition of various types of memories (Walker and Stickgold, 2004; Rauchs et al., 2005). Whereas the learning of simple procedural skills has been linked to stage 2 sleep (Peters et al., 2007), rapid eye movement (REM) sleep appears to be specifically implicated in the processing of complex cognitive tasks (Smith, 2001) and emotional memories (Stickgold et al., 2001; Wagner et al., 2001). In addition, the consolidation of episodic memories seems to be mainly dependent on REM sleep (Rauchs et al., 2004), and memories encoded while awake have been shown to be reactivated during subsequent sleep periods in both animals (Pavlides and Winson, 1989; Wilson and McNaughton, 1994) and humans (Maquet et al., 2000; Peigneux et al., 2004). This sleep-dependent processing is proposed to allow for a reinterpretation of the meaning of novel information and to facilitate its integration into a network of related memories (Paller and Voss, 2004). Furthermore, sleep appears to promote the mental restructuring involved in the discovery of hidden rules and consequently favor the gain of explicit knowledge that can influence behavior (Wagner et al., 2004).

The objective of the present study was to investigate the possibility that sleep contributes to the integration of new expectations and consequently influences the generation of expectation-mediated placebo effects. In group 1, strong expectations as to the efficacy of a placebo cream were generated before sleep by a conditioning manipulation, and expectations and analgesic effects were measured the following morning in a placebo testing block ( $12 \mathrm{~h}$ after 
conditioning). Placebo effects were then compared with those of a control daytime group (group 2) that comprised a $12 \mathrm{~h}$ postconditioning delay but no sleep episode. An additional night group was included (group 3), with subjects conditioned in the evening and tested both before and after sleep. Individual differences in expectations and analgesic effects reported in groups 1 and 3 were specifically investigated in relation to polysomnographic measures to test the hypothesis that expectation-mediated placebo effects are associated with changes in sleep architecture.

\section{Materials and Methods}

\section{Participants}

Thirty-eight healthy volunteers (22 females and 16 males, one left-handed) aged 20-35 years old $(23.42 \pm 0.47)$ were recruited on the campus of the University of Montréal and were alternately assigned to three groups. Groups were comparable on mean age $(23.7 \pm 2.3,23.2 \pm 3.5$, and $23.5 \pm 3.1$ years old, for groups 1,2 , and 3 ) and female/male proportion ( $7 / 12,8 / 13$, and $7 / 13$, for groups 1,2 , and 3 , respectively). Subjects reported no history of chronic pain, neurological, psychiatric, or sleep disorders, and no drug or medication consumption at the time of the experiment, except for contraceptive pills in 13 women. All women were tested during their follicular phase. Subjects were asked to abstain from caffeine and alcohol $24 \mathrm{~h}$ before and during the entire course of the study and reported regular sleep-wake rhythm (7-9 $\mathrm{h}$ of sleep per night, with morning awakening from 7:00 to 9:00 A.M.).

The study took place at the Centre for the Study of Sleep and Biological Rhythms at Sacré-Cœur Hospital (Montréal, Québec, Canada) where participants were greeted by the experimenter wearing a white lab coat. All instructions to participants followed a standardized script. Subjects were told that this experimental study aimed to evaluate the effect of sleep and circadian phase on the analgesic effects of a cream. The treatment was presented to participants as a topical cream that had proven effective in reducing pain in preliminary studies at other universities (Price et al., 1999). Participants were told that the exact composition of the cream could be revealed only at the end of the study. The experiment involved no risk for the subjects and was approved by the Hospital Ethics Committee. Participants signed a written consent form and were debriefed at the end of the study.

\section{Pain stimulation and experimental procedure}

Two sites of cutaneous stimulation, over each deltoid muscle, were delimitated for each participant. A $3 \mathrm{~cm}^{2}$ Peltier probe (NeuroSensory Analyser TSA-II; Medoc Ltd.) was used to induce contact heat pain (44$50^{\circ} \mathrm{C}$ ) from a baseline temperature of $32^{\circ} \mathrm{C}$. The stimulation protocol was similar to that developed by Price et al. (1999) and contained four different blocks of pain trials: familiarization, calibration, conditioning, and placebo testing. In the familiarization block, subjects were exposed to one trial each of $44,45,47$, and $49^{\circ} \mathrm{C}$ stimuli to become gradually accustomed to the stimuli. In the calibration block, series of stimulations were delivered to each arm using the ascending method of limits to determine the individually adjusted temperature to be used during the conditioning and experimental blocks for each participant, as described below.

At the beginning of the conditioning and placebo blocks, the same inert cream was applied to the control and treatment sites identified on each arm. The cream applied to the treated site (placebo site) was described as a topical analgesic, whereas the cream applied to the control site was described as an inert cream used to control for nonspecific effects of the vehicle compound of the analgesic cream. The placebo condition was assigned to the dominant arm in half the subjects. In both the conditioning and placebo test blocks, successive phasic stimuli were delivered with the temperature rising from baseline at a rate of $4^{\circ} \mathrm{C} / \mathrm{s}$ and maintained for $5 \mathrm{~s}$ at target intensity. The beginning of each stimulus was preceded by a $5 \mathrm{~s}$ auditory countdown, and successive stimulus onsets were separated by $60 \mathrm{~s}$ intervals to minimize the risk of local sensitization.

During the conditioning block, a sequence of eight stimuli was delivered to both the control and placebo site. Whereas the control site was stimulated at a moderate pain level $(50-60 / 100$ on the pain intensity scale) determined individually based on the first calibration block, the temperature of the stimuli applied to the placebo site was surreptitiously decreased by $2^{\circ} \mathrm{C}$ compared with the control site. This manipulation was intended to provide participants with an unambiguous experience of analgesia. In the placebo testing block, subjects received five thermal stimuli of the same predetermined moderate pain stimulation level on each arm.

\section{Experimental design}

The 38 participants were divided into three groups. In group $1(n=12)$ (Fig. $1 A$ ), familiarization and calibration blocks were performed at 8:00 P.M., and the conditioning procedure began at 9:00 P.M. Subjects were then prepared for nocturnal polysomnographic recording using standard electroencephalographic, electro-oculographic, and electromyographic measures. Lights were turned off at 11:00 P.M., and subjects were awakened at 7:00 A.M. the next morning. Placebo analgesia was evaluated $2 \mathrm{~h}$ after waking (9:00 A.M.). In group $2(n=13)$ (Fig. $1 B)$, familiarization and calibration blocks were performed at 8:00 A.M., conditioning manipulation started at 9:00 A.M., and placebo analgesia was tested in the evening at 9:00 P.M., $12 \mathrm{~h}$ after conditioning, to control for sleep-independent effects associated with the simple passage of time. Subjects in this group were free to perform their normal daily activities but were asked not to nap. Group $3(n=13)$ (Fig. 1C) followed a design similar to group 1, with the addition of an evening placebo test introduced $30 \mathrm{~min}$ after conditioning (placebo test 1 ) to evaluate placebo effects before sleep. This group was tested again the next morning (placebo test 2), as in group 1 . The addition of an evening placebo test in group 3 was used to test for the effect of the variable daytime delay between the conditioning manipulations and the evening placebo test ( $12 \mathrm{~h}$ in group 2; $30 \mathrm{~min}$ in group 3, test 1 ) and was expected to provide subjects with a more ambiguous experience of analgesia in the evening compared with conditioning alone (group 1). At the conclusion of the study (9:30 A.M. for groups 1 and 3, and 9:30 P.M. for group 2), another calibration block was administered on the control arm of all the participants.

\section{Dependent variables: pain ratings}

Subjective evaluations of pain intensity and unpleasantness were obtained using a $15 \mathrm{~cm}$ mechanical visual analog scale (VAS) (Price et al., 1983). Sensory VAS was anchored at the left by the descriptor "no pain sensation" and at the right by the descriptor "most intense pain imagin- 
able." Similarly, affective VAS was anchored at the left and right by the respective descriptors "not at all unpleasant" and "most unpleasant pain imaginable." These two scales were also used to rate expected and remembered pain. All ratings were linearly converted to a numerical value from 0 to 100.

Expected pain. Expected pain intensity and unpleasantness were prospectively acquired at the beginning of the conditioning and testing blocks. Participants were asked, "What do you expect the pain intensity/ unpleasantness to be without/with the analgesic cream?"

Concurrent pain. In the conditioning and testing blocks, participants were asked to rate the intensity and unpleasantness of the pain felt immediately after each stimulation.

Remembered pain. Approximately $2 \mathrm{~min}$ after the end of the placebo testing block, participants were asked to retrospectively rate the overall pain felt on the control and placebo sites: "Retrospectively, what was the overall pain intensity/unpleasantness felt without/with the analgesic cream?"

\section{Dependent variables: polysomnographic measures}

Sleep stage was monitored using standard polysomnographic methods. Electrodes were positioned at $\mathrm{Cz}, \mathrm{Fz}$, and $\mathrm{Oz}$ according to the International 10-20 System (Munday, 2005). Linked earlobes (A1 + A2) were used as a reference. Sleep stages were determined according to the guidelines of Rechtschaffen and Kales (1968) by experienced technicians blind to the aim of study and group assignment. All polysomnographic traces were reviewed by two independent technicians to extract the following dependent variables. The relative duration of the different sleep stages, expressed as the percentage of time spent in each stage, was calculated by dividing the total amount in minutes by the total sleep time. The number of atonic REM events was obtained from the number of $2 \mathrm{~s}$ milliepochs of REM sleep in which muscle activity was absent. Sleep latency corresponded to the time between lights off and the beginning of the first sleep episode, and sleep efficiency was calculated by dividing the duration of sleep by the overall duration of the sleep period (including nighttime awakenings). REM sleep latency corresponds to the delay between the first sleep event and the first REM sleep episode.

\section{Dependent variables: psychomotor vigilance task and questionnaires}

To control for potential fluctuations in vigilance and subjective sleepiness, a 10 min psychomotor vigilance task and the Karolinska Sleepiness Scale were administered to all subjects at the beginning and end of the study. In addition, the Beck Anxiety Inventory (BAI), the Pain Catastrophizing Scale (PCS), and the Life Orientation Test-Revised (LOT-R) were administered to all subjects.

\section{Statistical analysis}

Data are presented as mean \pm SEM. The placebo effect for expected, concurrent, and remembered pain intensity and unpleasantness ratings obtained in the placebo test performed $12 \mathrm{~h}$ after conditioning was analyzed using 3 (groups) $\times 2$ (treatments) ANOVAs. An additional ANOVA was performed to compare the evening placebo response of group 3 (placebo test 1 ) and group 2 to assess the simple effect of the delay between the conditioning block and the placebo test (group 3, test 1: 30 min; group 2: $12 \mathrm{~h}$ ). Analgesia was calculated as the difference (control placebo) in VAS ratings of intensity and unpleasantness for expected, concurrent, and remembered pain. Placebo analgesia did not vary with the laterality of the placebo test (dominant vs nondominant arm; all $p$ values $>0.05$ ), and this factor was not considered further in the analysis. Analgesia scores were used to calculate Pearson's $r$ correlations and estimate explained variance $\left(R^{2}\right)$. Additionally, independent $t$ tests were used when appropriate, and the nonparametric Kruskal-Wallis test was used to assess score differences in questionnaires. Statistical analyses were performed with SPSS 16.0, and global coincidence tests were performed with Prism 5.0. The global coincidence test allows the comparison of entire curves (slopes and intercepts) using an $F$ test. To do so, the sumof-square and degrees of freedom from the best fit of each curve is added and compared with a combined model in which the slopes and intercepts are shared among the datasets. All statistical tests were performed at

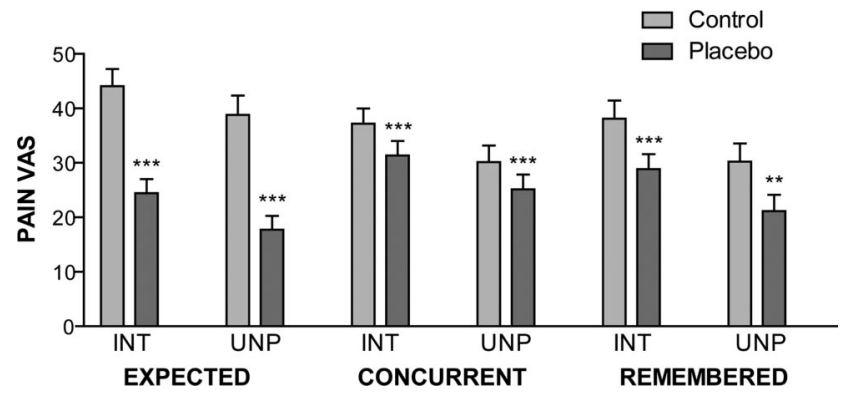

Figure 2. Expected, concurrent, and remembered pain intensity (INT) and unpleasantness (UNP) measured $12 \mathrm{~h}$ after conditioning on control and placebo sites for all 38 subjects. Paired $t$ test; ${ }^{* *} p<0.01,{ }^{* * *} p<0.001$.

$\alpha=0.05$, and multiple comparisons were accounted for using Bonferroni's adjustments. Effect sizes for main effects and interactions (ANOVAs) were calculated using partial $\eta$ squared $\left(\eta_{\mathrm{p}}^{2}\right)$, and pairwise contrasts were calculated using Cohen's $d$ with Hedges-bias correction (Cohen, 1988).

\section{Results}

\section{Effects of conditioning}

Because the experimental design involved pain stimuli applied in the morning and evening, two calibration blocks were performed for all 38 subjects to assess potential variations in pain with regards to circadian phase. The results show that the temperature required to induce moderate pain was similar in the morning and evening calibration blocks $\left(48.5 \pm 0.2\right.$ vs $48.4 \pm 0.2^{\circ} \mathrm{C} ; F_{(1,35)}=$ $0.355, p=0.56)$. In the conditioning block, the stimulus intensities used for each individual were adjusted based on the first calibration block to generate moderate pain in control condition (average of $50.7 \pm 3.3$ VAS units on the pain intensity scale). The overall mean temperatures used in the conditioning block for control and placebo conditions were $48.7 \pm 0.1$ and $46.7 \pm 0.1^{\circ} \mathrm{C}$, respectively. Expected pain intensity reductions (control - placebo) induced by the placebo cream before conditioning procedures were comparable across groups (groups 1, 2, 3: $16.6 \pm 3.2$ vs $14.2 \pm 2.7$ vs $18.4 \pm 3.6$ VAS units; $\left.F_{(2,35)}=0.44, p=0.65\right)$. Similarly, the three groups reported comparable levels of analgesia in concurrent pain intensity ratings during the conditioning manipulations (groups 1, 2, 3: $19.0 \pm 4.2$ vs $21.8 \pm 3.5$ vs $20.2 \pm$ 4.1 VAS units; $\left.F_{(2,35)}=0.13, p=0.88\right)$. Comparable results were found for expected pain unpleasantness reductions $\left(F_{(2,35)}=\right.$ $0.78, p=0.47)$ and concurrent pain unpleasantness reductions $\left(F_{(2,35)}=0.10, p=0.90\right)$ during the conditioning manipulations. These results indicate that, before any manipulation, all subjects displayed similar initial expectations toward the proposed treatment and that the conditioning procedures induced a comparable experience of pain relief across the three groups.

\section{Assessment of placebo effects and mediating influence of expectations}

For all 38 participants, placebo analgesia was evaluated $12 \mathrm{~h}$ after conditioning. Expected, concurrent, and remembered pain intensity and unpleasantness ratings for control and placebo sites are summarized in Figure 2. The results show that, $12 \mathrm{~h}$ after conditioning, subjects expected a substantial reduction in pain intensity after placebo treatment $\left(F_{(1,35)}=108.4, p<0.001, \eta_{p}^{2}=0.756\right)$, which was comparable across groups (main effect of group, $F_{(2,35)}=0.54, p=0.59$; interaction, $\left.F_{(2,35)}=0.45, p=0.64\right)$. Similar results were found for expected pain unpleasantness reductions (main effect of placebo treatment, $F_{(1,35)}=63.46, p<$ 
Table 1. Concurrent and remembered placebo effects and correlations between expected relief and concurrent and remembered pain reductions across groups and placebo tests

\begin{tabular}{|c|c|c|c|c|c|}
\hline & $n$ & $\begin{array}{l}\text { Concurrent analgesia, } \\
\text { Cohen's d (Cl) }\end{array}$ & $\begin{array}{l}\text { Remembered analgesia, } \\
\text { Cohen's } d(\mathrm{Cl})\end{array}$ & $\begin{array}{l}\text { Expected } \times \text { concurrent; } \\
\text { Pearson's } r\end{array}$ & $\begin{array}{l}\text { Expected } \times \text { remembered; } \\
\text { Pearson's } r\end{array}$ \\
\hline \multicolumn{6}{|l|}{ Pain intensity } \\
\hline Group 1 (overnight delay; morning test) & 12 & $1.16(0.29-2.02)$ & $1.17(0.31-2.04)$ & $0.88^{* * *}$ & $0.86^{* * *}$ \\
\hline Group 2 (daytime delay; evening test) & 13 & $0.93(0.12-1.74)$ & $1.34(0.49-2.19)$ & $0.61^{*}$ & $0.66^{*}$ \\
\hline Group 3, test 1 (no delay; evening test) & 13 & $0.80(0.00-1.60)$ & $0.89(0.08-1.69)$ & $0.49^{\dagger}$ & 0.12 \\
\hline Group 3, Test 2 (overnight delay; morning test) & 13 & $0.65(-0.14$ to 1.43$)$ & $0.54(-0.24$ to 1.32$)$ & -0.23 & -0.43 \\
\hline \multicolumn{6}{|l|}{ Pain unpleasantness } \\
\hline Group 1 (overnight delay; morning test) & 12 & $0.95(0.11-1.80)$ & $0.93(0.09-1.77)$ & $0.83^{* * *}$ & $0.87^{* * *}$ \\
\hline Group 2 (daytime delay; evening test) & 13 & $0.89(0.08-1.69)$ & $1.29(0.45-2.14)$ & $0.68^{* *}$ & $0.78^{* *}$ \\
\hline Group 3, test 1 (no delay; evening test) & 13 & $0.57(-0.21$ to 1.36$)$ & $1.30(0.45-2.14)$ & 0.31 & 0.05 \\
\hline Group 3, test 2 (overnight delay; morning test) & 13 & $0.54(-0.24$ to 1.32$)$ & $0.41(-0.36$ to 1.19$)$ & -0.35 & $-0.49^{\dagger}$ \\
\hline
\end{tabular}

Cohen's $d$ with Hedges-bias correction was used to evaluate effect size, and Pearson's correlation coefficients $(r)$ were established between expected, concurrent, and remembered analgesia scores. Analgesia scores correspond to the difference between pain ratings at the control and placebo stimulation sites. Cl, Confidence interval. ${ }^{*} p<0.05,{ }^{* *} p<0.01,{ }^{* * *} p<0.001,{ }^{\dagger} p=0.09$.

$0.001, \eta_{\mathrm{p}}^{2}=0.645$; main effect of group, $F_{(2,35)}=0.01, p=0.99$; interaction, $\left.F_{(2,35)}=0.14, p=0.87\right)$.

ANOVA of concurrent pain intensity during the testing block revealed a main effect of placebo treatment $\left(F_{(1,35)}=15.68, p<\right.$ $\left.0.001, \eta_{\mathrm{p}}^{2}=0.309\right)$, with no significant effect of, or interaction with, groups (main effect of group, $F_{(2,35)}=0.05, p=0.95$; interaction, $\left.F_{(2,35)}=0.54, p=0.587\right)$. Likewise, the application of a placebo considerably reduced concurrent pain unpleasantness $\left(F_{(1,35)}=11.55, p<0.002, \eta_{\mathrm{p}}^{2}=0.248\right)$, with no effect of, or interaction with, groups (main effect of group, $F_{(2,35)}=0.19, p=$ 0.829 ; interaction, $\left.F_{(2,35)}=0.36, p=0.697\right)$. These effects corresponds to a mean decrease of $13.5 \pm 3.9 \%$ in concurrent pain intensity $(p=0.001)$ and a $14.3 \pm 4.5 \%$ decrease in concurrent pain unpleasantness $(p=0.003)$. Similarly, retrospective pain intensity evaluations revealed significant analgesia induced by the placebo treatment $\left(F_{(1,35)}=17.97, p<0.001, \eta_{\mathrm{p}}^{2}=0.339\right)$, with no effect of, or interaction with, groups (main effect of group, $F_{(2,35)}=0.19, p=0.829$; interaction, $F_{(2,35)}=0.72, p=$ $0.495)$. Retrospective pain unpleasantness showed a similar pattern (main effect of placebo treatment, $F_{(1,35)}=11.37, p=0.002$, $\eta_{\mathrm{p}}^{2}=0.245$; main effect of group, $F_{(2,35)}=0.53, p=0.592$; interaction, $\left.F_{(2,35)}=0.87, p=0.426\right)$. This demonstrates that, $12 \mathrm{~h}$ after the conditioning manipulations, a significant placebo effect was observed across all three groups.

In group 3, placebo effects were also measured in the evening, $30 \mathrm{~min}$ after the completion of the conditioning block. When compared with the placebo effects also measured in the evening for subjects in group 2 but $12 \mathrm{~h}$ after conditioning, no significant differences were noted between groups $\left(F_{(1,24)}=0.50 ; p=0.49\right)$, and the significant effect of placebo treatment $\left(F_{(1,24)}=9.64, p=\right.$ $0.005)$ did not show any interaction with groups $\left(F_{(1,24)}=0.30\right.$, $p=0.59)$. These results indicate that the daytime delay between the conditioning block and the placebo test did not alter the magnitude of placebo response.

Table 1 summarizes the placebo responses measured during the testing block in each group. In group 1, a sleep episode was present between the conditioning procedures and the testing block, and subjects in this group displayed a strong analgesic effect $12 \mathrm{~h}$ after conditioning for both concurrent and remembered relief, with expectations predicting as much as 77 and $74 \%$ of pain intensity reductions $\left(R^{2}\right)$. In group 2 , the experimental manipulations were performed during the daytime, and subjects were tested $12 \mathrm{~h}$ after conditioning without having slept. A strong placebo effect was also reported, but the predictive value of expectations on concurrent and remembered placebo response decreased to 38 and $44 \%$, respectively. These results suggest that sleep may contribute to the consolidation of the mediating effect of expectations on placebo responses. However, because the lack of EEG fitting in the control daytime group 2 might have prevented the reinforcement of expectations by exposure to a more elaborate experimental protocol, the difference between the initial expectations and the expected reliefs measured $12 \mathrm{~h}$ later was assessed. The results show that expectations in both groups were increased to a similar extend by the experimental manipulations (main effect, $F_{(1,23)}=8.07, p=0.009$; interaction, $F_{(1,23)}=0.24$, $p=0.63$; main effect of group, $\left.F_{(1,23)}=0.80, p=0.38\right)$, which suggest that the exposure to EEG in group 1 did not further enhance expectations when compared with group 2 .

Subjects in group 3 were tested both before and after sleep, with a moderate level of repeatability $(r=0.52, p=0.066)$, consistent with previous studies (Wager et al., 2004). The first test was conducted in the evening $30 \mathrm{~min}$ after conditioning and showed the presence of an analgesic effect of placebo treatment, although expectations were only marginally associated with $24 \%$ of the concurrent pain intensity reductions. These results are consistent with the effects measured in group 2 and suggest that, when placebo analgesia was evaluated in the evening after either a short or long delay without the presence of sleep, expectations accounted for $24-38 \%$ of the placebo effect observed in concurrent pain intensity reports. These expectation-related responses contrasted with the effects observed in group 1 .

The second placebo-test block in group 3 was performed in the morning, $12 \mathrm{~h}$ after conditioning. The results show a moderate placebo effect in the morning for both concurrent and remembered pain intensity and unpleasantness reductions (Table 1). In addition, placebo-related expectations did not significantly predict changes in concurrent or remembered pain. When compared with subjects in group 1 who only underwent the conditioning block before sleep, the global coincidence test revealed that the correlations between expected relief and pain intensity reductions as well as pain unpleasantness reductions measured in the morning in those two groups were significantly different (concurrent intensity, $F_{(2,21)}=7.02, p=$ 0.0046; remembered intensity, $F_{(2,21)}=9.09, p=0.0014$; concurrent unpleasantness, $F_{(2,21)}=7.62, p=0.0033$; remembered unpleasantness, $\left.F_{(2,21)}=12.58, p=0.0003\right)$. These results indicate that the presence of an evening testing block in addition to the conditioning manipulations before sleep hinders the establishment of expectation-mediated analgesic effects the following morning and changes the relationship between expected relief and placebo analgesia. 
Table 2. Control sleep parameters for the 25 subjects recorded with polysomnography

\begin{tabular}{lllll}
\hline & $\begin{array}{l}\text { Sleep latency } \\
(\mathrm{min})\end{array}$ & $\begin{array}{l}\text { Sleep duration } \\
(\mathrm{min})\end{array}$ & $\begin{array}{l}\text { Number of } \\
\text { awakenings }\end{array}$ & $\begin{array}{l}\text { Sleep } \\
\text { efficiency (\%) }\end{array}$ \\
\hline Group 1 & $12.93 \pm 4.39$ & $441.91 \pm 8.54$ & $33.42 \pm 3.06$ & $92.73 \pm 1.09$ \\
Group 3 & $12.74 \pm 2.94$ & $434.56 \pm 7.01$ & $26.15 \pm 2.38$ & $93.93 \pm 1.03$ \\
\hline
\end{tabular}

Control parameters: vigilance, sleep quality, and questionnaires

A 10 min psychomotor vigilance task administered at the beginning and end of the study revealed no significant differences in vigilance between the three groups (main effect of group for mean reaction time, $\left.F_{(2,34)}=1.12, p=0.34\right)$, nor between the time of testing $\left(F_{(1,34)}=0.54, p=0.47\right.$; interaction, $F_{(2,34)}=0.04$, $p=0.96)$. Subjective sleepiness assessed by the Karolinska Sleepiness Scale showed comparable results at the beginning of the study $\left(H_{(2)}=0.989, p=0.610\right.$, Kruskal-Wallis test), but group 1 reported less sleepiness at the end of the experiment $\left(H_{(2)}=\right.$ $6.617, p=0.037$, Kruskal-Wallis test). To verify that sleep quality was comparable between the two night groups, different sleep parameters were assessed, as reported in Table 2. Overall, there were no differences in sleep latency, sleep duration, number of awakenings, or sleep efficiency between group 1 and group 3 (all $p$ values $>0.05$, independent $t$ test). In addition, the 25 participants who slept in the laboratory (groups 1 and 3) spent $57.7 \pm 1.6 \%$ of their total sleep time in stage 2 sleep, $15.2 \pm$ $1.4 \%$ in slow-wave sleep, and $20.3 \pm 0.9 \%$ in REM sleep. A normal $7.5 \mathrm{~h}$ sleep period in young healthy adults generally constitutes $45-55 \%$ stage 2 sleep, $13-23 \%$ slow-wave sleep, and $20-25 \%$ REM sleep, with wakefulness accounting for $\sim 5 \%$ (Kryger et al., 2005).

Psychological measures of anxiety, catastrophizing, and optimism were also compiled for each subject. Scores from the Beck Anxiety Inventory, the Pain Catastrophizing Scale, or the Life Orientation Test-Revised did not reveal any differences between the three groups (Kruskal-Wallis test; BAI, $H_{(2)}=0.744, p=$ 0.69 ; PCS, $H_{(2)}=0.462, p=0.79$; LOT-R, $H_{(2)}=2.785, p=$ $0.25)$. Furthermore, they did not show any significant correlation with any measures of placebo analgesia or sleep variables, either as a hole or within each group (Spearman's test, all $p$ values $>0.05$ ). These results suggest that the personality traits assessed here did not have a significant mediating effect on either sleep or placebo susceptibility, nor could they explain potential groups differences.

\section{Effect of placebo manipulations on sleep architecture}

Comparative analysis of polysomnographic recordings between the two night groups revealed that subjects in group 1 displayed a higher proportion of stage 2 sleep then group $3(61.4 \pm 2.0$ vs $\left.54.3 \pm 1.9 \% ; F_{(1,21)}=6.18, p=0.021\right)$ and spent a lesser proportion of their total sleep time in REM sleep (18.7 \pm 1.5 vs $21.7 \pm$ $1.1 \% ; F_{(1,21)}=4.69, p=0.042$ ) (Fig. 3). Because the placebo manipulations were designed to alter expectations and placebo responses, their influence on sleep profiles was also analyzed by dividing the groups into placebo responders $(n=12)$ and nonresponders $(n=13)$ based on a $20 \%$ decrease in concurrent pain intensity ratings (Zubieta et al., 2005), which here corresponded to a large effect size at $d=0.82$, and divided the experimental groups close to the median. A significant interaction was observed, whereby only placebo responders in group 1 showed a significant reduction in the percentage of total sleep time spent in $\operatorname{REM}\left(F_{(1,21)}=6.61, p=0.018\right.$ ) (Fig.

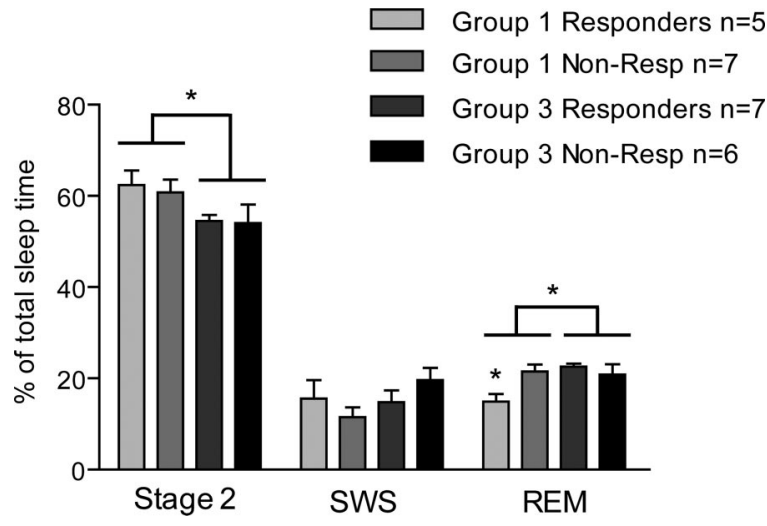

Figure 3. Sleep architecture of placebo responders and nonresponders of groups 1 and 3 . Subjects in group 1 displayed higher proportions of stage 2 sleep and smaller proportions of REM sleep. The reduction in REM sleep was specific to placebo responders in group 1. ANOVA, ${ }^{*} p<$ 0.05. SWS, Slow-wave sleep.

3). This REM sleep decrease corresponds to a remarkable 30.2 min difference from an average of $95.5 \mathrm{~min}$ for the other subgroups. Additional analysis revealed that the placebo response influenced REM sleep latency correspondingly, in which placebo responders had significantly longer REM latency than nonresponders $\left(125.96 \pm 12.18\right.$ vs $88.11 \pm 11.57 \mathrm{~min} ; F_{(1,21)}=5.08$, $p=0.035)$. These results demonstrate that profile differences in evening exposure to the placebo significantly altered sleep architecture and that the reduction in REM sleep observed in placebo responders in group 1 is related to extended REM sleep latency.

To further explore the relationship between REM sleep, expectation, and placebo analgesia, we correlated the morning analgesia scores with the percentage of REM sleep in all subjects for each of the two night groups (Fig. 4). In group 1, expected, concurrent, and remembered analgesia for both intensity and unpleasantness were negatively correlated with the proportion of REM sleep (trend at $r=-0.521, p=0.082$ for concurrent pain intensity relief, all other $p$ values $<0.05$ ) (Pearson's $r$ and $p$ values are reported in Fig. 4). In this condition, it appears that subjects who spent a lesser proportion of their total sleep time in REM sleep expected, experienced, and remembered higher treatmentinduced relief in the placebo test performed the next morning. Conversely, REM sleep measured in subjects in group 3 was positively correlated with the analgesia expected in the evening (intensity, $r=0.626, p=0.022$; unpleasantness, $r=0.604, p=$ 0.029 ) and morning (Fig. 4) placebo tests. However, in this group, changes in REM sleep were not significantly related to either concurrent or remembered placebo analgesia in the morning (Fig. 4) and only marginally associated with concurrent placebo analgesia measured in the preceding evening (intensity, $r=0.519, p=0.069$; unpleasantness, $r=0.525, p=0.065$ ). Similar results were found for both groups when analgesia scores were correlated with the number of atonic REM events (supplemental Fig. 1, available at www.jneurosci.org as supplemental material). In addition, global coincidence analysis revealed that the correlations established between expected analgesia in the morning and REM sleep in groups 1 and 3 were significantly different (intensity, $F_{(2,21)}=5.21, p=0.0145$; unpleasantness, $\left.F_{(2,21)}=8.65, p=0.0018\right)$. These findings indicate that the relation between REM sleep and both expectations and placebo analgesia experienced in the morning are critically influenced by previous experience with the treatment in the preceding evening. 


\section{A Group 1}
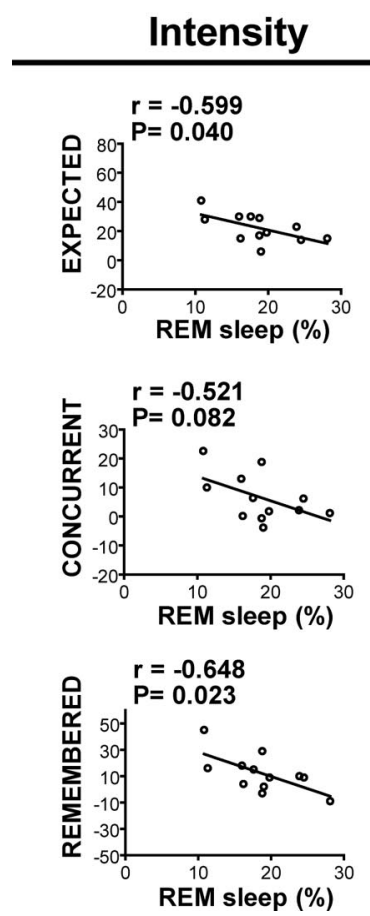

Unpleasantness
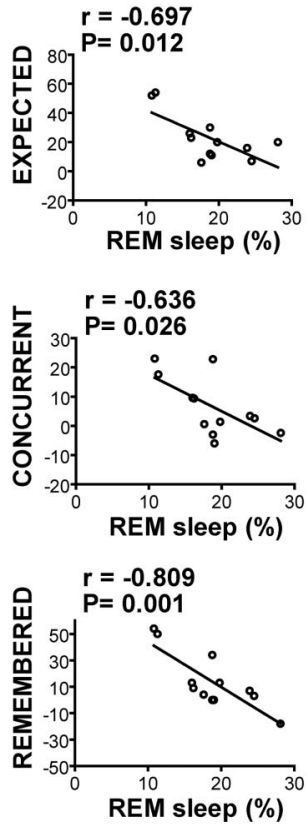

B Group 3 test 2

Intensity Unpleasantness
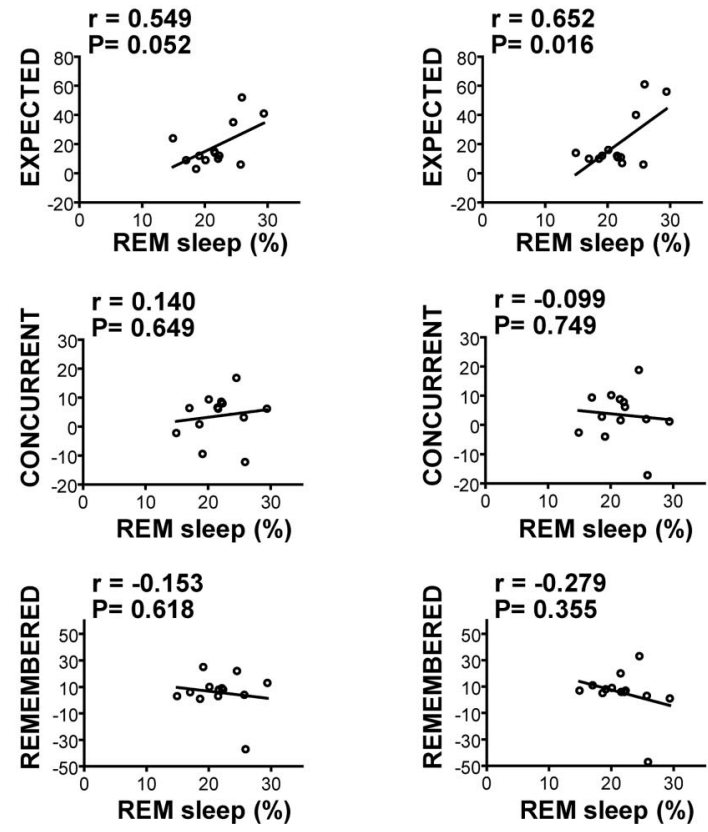

Figure 4. Correlation of expected, concurrent, and remembered analgesia (control-placebo) in pain intensity and unpleasantness with percentage REM sleep measured in the night before the testing block in group $1(\boldsymbol{A})$ and group $3(\boldsymbol{B})$, test 2 (morning). Pearson's $r$ and corresponding $p$ values are reported on the graphs.

\section{Discussion}

Effect of sleep on the integration of new expectations

In the present study, we adapted a placebo analgesia induction protocol used previously by others (Price et al., 1999; Petrovic et al., 2002; Wager et al., 2004, 2007; Bingel et al., 2006) to investigate the effect of sleep on the development of expectation-related placebo responses. Suggestion of pain relief and sensory conditioning were used to create analgesic expectations toward a placebo cream, and placebo responses were measured $12 \mathrm{~h}$ after conditioning. As shown previously (Montgomery and Kirsch, 1997; Price et al., 1999; Benedetti et al., 2003), expectations were a significant mediator of the analgesic effects, but their relative contribution varied considerably when the postconditioning delay included a sleep period. In this condition, expectations were tightly correlated with placebo analgesia and predicted as much as $77 \%$ of the effect. The acquisition of expectations leading to changes in perception can be viewed in the general framework of episodic memory formation in which new information is acquired, consolidated, and recalled. In the present experiment, expectations of relief induced experimentally were gradually integrated into an existing network of personal beliefs and past experience and were evoked in subsequent testing. During sleep, the reactivation of memory traces of recent waking events have been demonstrated in both animals (Wilson and McNaughton, 1994; Louie and Wilson, 2001) and humans (Maquet et al., 2000; Peigneux et al., 2004), and this process is proposed to consolidate various types of learning. Because placebo effects can be at least partly attributed to learning effects (Voudouris et al., 1990; Colloca et al., 2008), sleep might enhance the integration of complex experiences and reinforce the impact of expectations on subsequent perceptions. Our results suggest that sleep-dependent processing of the information acquired during conditioning enhanced the integration of new expectations, which in turn strongly modulated experienced relief. In addition, because the magnitude of the analgesic effect was not significantly different between groups, our results indicate that sleep might act specifically on the expectation-mediated component of the analgesic effect by strengthening the association between expectations and analgesia, although other independent factors might also contribute to the overall extent of the effect.

\section{Expectations and REM sleep}

Because the mediating role of expectations in placebo analgesia appeared to be facilitated by the presence of a sleep period, changes in the physiological features of postconditioning sleep could potentially reflect this process. In group 1 , the exposure to treatment before sleep was manipulated in the conditioning phase to generate a convincing experience of analgesia, which translated into a robust expectation-mediated placebo response the next morning. In this condition, reduction in REM sleep was associated with stronger expectations of pain relief and stronger placebo effects the next morning. Consistently, placebo responders specifically exhibited the greatest reduction in REM sleep (and correspondingly longer REM sleep latency) compared with nonresponders and compared with the normal sleep architecture generally observed in young healthy subjects, as described previously in our laboratory and others (Aslan et al., 2002; Shaw et al., 2005). Moreover, the decrease in REM sleep not only reflected individual expectations but also predicted concurrent pain unpleasantness reductions and remembered relief in the morning placebo test. Previous preliminary data in healthy individuals revealed a relationship between individual variation in REM sleep and central pain-modulatory processes, such that subjects who normally express a greater percentage of REM sleep have heightened central sensitivity to pain through increased supraspinal pain facilitation (Smith et al., 2005). This is consistent 
with the present results demonstrating stronger expectationrelated pain relief in individuals showing reduced REM sleep.

Sleep architecture changes have been observed in the night after training in a variety of tasks (for review, see Smith, 1995; Rauchs et al., 2005), but, to our knowledge, the learned association between a placebo treatment and analgesia has never been studied in relation to sleep. Nevertheless, the consolidation of episodic memories and the reprocessing of complex cognitive tasks have been linked previously with REM sleep alterations (Smith, 2001; Rauchs et al., 2004). In the current study, when positive expectations were accompanied by a consistent sensory experience before sleep (group 1), placebo responders specifically displayed a reduction in the amount of time they spent in REM sleep. Because high expectations likely reflect a concordance between the new episode and previous beliefs, REM sleep reductions could reflect a limited need to seek implicit information and restructuration. Indeed, processes occurring during REM sleep have been proposed to assist neurocognitive searches for novel interpretations (Maquet et al., 2003) and to be involved in the discovery of hidden associative rules (Walker et al., 2002). For example, in a paradigm of implicit sequence learning, REM sleep has been shown to be particularly important for the acquisition of the implicit rule rather than the motor component, which suggests that REM sleep in particular is involved in the consolidation of implicitly acquired complex relationships (Peigneux et al., 2003). In this view, if the new experience is coherent with previously consolidated beliefs, its integration into a network of older related memories could be facilitated, and the requirement for cognitive reappraisal during REM sleep would therefore be diminished. Conversely, in subjects in group 3, the presence of the conditioning manipulations and a testing block generated a more inconsistent sensory experience before sleep. Notwithstanding, high expectations of relief were still observed in some participants, but, contrary to the first night group, the results indicate that subjects who anticipated more relief spent a higher percentage of their sleep time in REM sleep. In light of the proposed role of REM in the reprocessing of implicit information, this positive relation suggests that REM sleep contributed to the preservation of positive expectations despite the implicit sensorial conflict. Interestingly, in this context, expectations did not emerge as an important mediator of the analgesic effects measured the next morning, and REM sleep did not predict placebo effects. This suggests that, when sensory input is inconsistent, sleep might promote the dissociation of expectations from the observed placebo effect and that the residual morning placebo response observed in group 3 may reflect a persistent effect independent of both expectations and changes in REM sleep.

Together, these findings show that the relative duration of REM sleep can predict expectation-mediated analgesic effects but that subtle differences in previous experiences can dramatically alter this relationship. The degree of certainty associated with an expectation is another important mediating factor that triggers different emotional states, which can in turn significantly influence pain perception (Ploghaus et al., 2003). Whereas certainty that a particular aversive event is impending reduces pain sensitivity, uncertain expectations may generate anxiety-mediated hyperalgesia (Ploghaus et al., 2001). In the current study, it is possible that the inconsistent sensory inputs delivered to participants before sleep in group 3 may have generated a mediating emotional state that necessitated additional REM-dependent cognitive reprocessing, particularly in subjects displaying high expectations. This is in line with previous findings that the strength and direction of the relationship between waking expe- riences and REM sleep can be moderated by situational and emotional factors (Germain et al., 2003). However, in the present study, the psychological variables assessed (anxiety, pain catastrophizing, and optimism) could not explain the effects observed, suggesting that the mediating emotional states induced by the placebo manipulations were not associated with such traits but rather with a contextual transient emotional set. Future investigations using direct measures of certainty and anticipatory emotions generated by discordant cognitive information and sensory inputs are needed to better understand the exact mindset of subjects exposed to a placebo treatment and how it influences sleep architecture.

In addition to the effects observed on REM sleep, stage 2 sleep was also altered by the evening placebo manipulations. Along with the decrease in REM sleep, subjects exposed solely to the conditioning block before sleep displayed an increase in stage 2 sleep. Although stage 2 sleep has mainly been associated with the acquisition of simple procedural skills (Peters et al., 2007), its involvement in the consolidation of episodic memories has also been proposed (Rauchs et al., 2005; Fogel and Smith, 2006). In this view, whereas REM sleep might have been implicated in the reprocessing of implicit information associated with the episode, stage 2 sleep could underlie the maintenance of a different aspect of the experience. Alternatively, because stage 2 sleep did not correlate with placebo analgesia measured in the subsequent testing blocks (not reported in detail here), the increase in stage 2 sleep observed in this group might reflect a simple compensatory mechanism that counterbalanced the decrease in REM sleep. Because the current experimental manipulations admittedly involved many types of memory systems, the interactions with sleep were likely to be complex, and different sleep stages could have been implicated in the processing of various dimensions of the task.

\section{Clinical relevance}

Although the evidence linking placebo responses and sleep architecture is scarce, an interesting study demonstrated that patients with panic disorders responding to either pharmacological or placebo treatments exhibited normalized sleep patterns, characterized by a reduction in the percentage of REM sleep (Baker et al., 2003). Because anxiety is believed to be one of the factors involved in placebo effects (Benedetti et al., 2005; Price et al., 2008), the authors postulated that the reduction of REM sleep observed after placebo administration reflected diminished anxiety and that the amount of REM sleep after exposure to a treatment might represent a correlate of therapeutic response. Interestingly, the current investigation, performed in healthy individuals, revealed a very similar reduction in REM sleep in placebo responders to that described by Baker et al. (2003) (14.9 vs $14.7 \%)$. Together, these findings suggest that the generation of a transient positive emotional state induced by relief expectation may significantly alter sleep profiles and that reduction in REM sleep might represent a correlate of expectation-mediated therapeutic responses.

\section{References}

Aslan S, Isik E, Cosar B (2002) The effects of mirtazapine on sleep: a placebo controlled, double-blind study in young healthy volunteers. Sleep 25:677-679.

Baker B, Khaykin Y, Devins G, Dorian P, Shapiro C, Newman D (2003) Correlates of therapeutic response in panic disorder presenting with palpitations: heart rate variability, sleep, and placebo effect. Can J Psychiatry 48:381-387.

Benedetti F, Pollo A, Lopiano L, Lanotte M, Vighetti S, Rainero I (2003) 
Conscious expectation and unconscious conditioning in analgesic, motor, and hormonal placebo/nocebo responses. J Neurosci 23:4315-4323.

Benedetti F, Mayberg HS, Wager TD, Stohler CS, Zubieta JK (2005) Neurobiological mechanisms of the placebo effect. J Neurosci 25:10390-10402.

Bingel U, Lorenz J, Schoell E, Weiller C, Büchel C (2006) Mechanisms of placebo analgesia: rACC recruitment of a subcortical antinociceptive network. Pain 120:8-15.

Cohen J (1988) Statistical power analysis for the behavioral sciences, Ed 2. Hillsdale, NJ: Erlbaum.

Colloca L, Benedetti F (2006) How prior experience shapes placebo analgesia. Pain 124:126-133.

Colloca L, Sigaudo M, Benedetti F (2008) The role of learning in nocebo and placebo effects. Pain 136:211-218.

Fogel SM, Smith CT (2006) Learning-dependent changes in sleep spindles and Stage 2 sleep. J Sleep Res 15:250-255.

Germain A, Buysse DJ, Ombao H, Kupfer DJ, Hall M (2003) Psychophysiological reactivity and coping styles influence the effects of acute stress exposure on rapid eye movement sleep. Psychosom Med 65:857-864.

Koyama T, McHaffie JG, Laurienti PJ, Coghill RC (2005) The subjective experience of pain: where expectations become reality. Proc Natl Acad Sci U S A 102:12950-12955.

Kryger MH, Roth T, Dement WC (2005) Principles and practice of sleep medicine, Ed 4. Philadelphia: Elsevier Saunders.

Louie K, Wilson MA (2001) Temporally structured replay of awake hippocampal ensemble activity during rapid eye movement sleep. Neuron 29:145-156.

Maquet P, Laureys S, Peigneux P, Fuchs S, Petiau C, Phillips C, Aerts J, Del Fiore G, Degueldre C, Meulemans T, Luxen A, Franck G, Van Der Linden M, Smith C, Cleeremans A (2000) Experience-dependent changes in cerebral activation during human REM sleep. Nat Neurosci 3:831-836.

Maquet P, Smith C, Stickgold R (2003) Sleep and brain plasticity. Oxford: Oxford UP.

Montgomery GH, Kirsch I (1997) Classical conditioning and the placebo effect. Pain 72:107-113.

Munday JA (2005) Instrumentation and electrode placement. Respir Care Clin N Am 11:605-615, viii.

Paller KA, Voss JL (2004) Memory reactivation and consolidation during sleep. Learn Mem 11:664-670.

Pavlides C, Winson J (1989) Influences of hippocampal place cell firing in the awake state on the activity of these cells during subsequent sleep episodes. J Neurosci 9:2907-2918.

Peigneux P, Laureys S, Fuchs S, Destrebecqz A, Collette F, Delbeuck X, Phillips C, Aerts J, Del Fiore G, Degueldre C, Luxen A, Cleeremans A, Maquet P (2003) Learned material content and acquisition level modulate cerebral reactivation during posttraining rapid-eye-movements sleep. Neuroimage 20:125-134.

Peigneux P, Laureys S, Fuchs S, Collette F, Perrin F, Reggers J, Phillips C, Degueldre C, Del Fiore G, Aerts J, Luxen A, Maquet P (2004) Are spatial memories strengthened in the human hippocampus during slow wave sleep? Neuron 44:535-545.

Peters KR, Smith V, Smith CT (2007) Changes in sleep architecture following motor learning depend on initial skill level. J Cogn Neurosci 19:817-829.

Petrovic P, Kalso E, Petersson KM, Ingvar M (2002) Placebo and opioid analgesia- imaging a shared neuronal network. Science 295:1737-1740.

Ploghaus A, Narain C, Beckmann CF, Clare S, Bantick S, Wise R, Matthews PM,
Rawlins JN, Tracey I (2001) Exacerbation of pain by anxiety is associated with activity in a hippocampal network. J Neurosci 21:9896-9903.

Ploghaus A, Becerra L, Borras C, Borsook D (2003) Neural circuitry underlying pain modulation: expectation, hypnosis, placebo. Trends Cogn Sci 7:197-200.

Price DD, McGrath PA, Rafii A, Buckingham B (1983) The validation of visual analogue scales as ratio scale measures for chronic and experimental pain. Pain 17:45-56.

Price DD, Milling LS, Kirsch I, Duff A, Montgomery GH, Nicholls SS (1999) An analysis of factors that contribute to the magnitude of placebo analgesia in an experimental paradigm. Pain 83:147-156.

Price DD, Finniss DG, Benedetti F (2008) A comprehensive review of the placebo effect: recent advances and current thought. Annu Rev Psychol 59:565-590.

Rauchs G, Bertran F, Guillery-Girard B, Desgranges B, Kerrouche N, Denise P, Foret J, Eustache F (2004) Consolidation of strictly episodic memories mainly requires rapid eye movement sleep. Sleep 27:395-401.

Rauchs G, Desgranges B, Foret J, Eustache F (2005) The relationships between memory systems and sleep stages. J Sleep Res 14:123-140.

Rechtschaffen A, Kales A (1968) A Manual of standardized terminology, techniques and scoring system for sleep stages of human subjects. Washington, DC: Public Health Service, United States Government Printing Office.

Shaw IR, Lavigne G, Mayer P, Choinière M (2005) Acute intravenous administration of morphine perturbs sleep architecture in healthy pain-free young adults: a preliminary study. Sleep 28:677-682.

Smith C (1995) Sleep states and memory processes. Behav Brain Res 69:137-145.

Smith C (2001) Sleep states and memory processes in humans: procedural versus declarative memory systems. Sleep Med Rev 5:491-506.

Smith MT, Edwards RR, Stonerock GL, McCann UD (2005) Individual variation in rapid eye movement sleep is associated with pain perception in healthy women: preliminary data. Sleep 28:809-812.

Stickgold R, Hobson JA, Fosse R, Fosse M (2001) Sleep, learning, and dreams: off-line memory reprocessing. Science 294:1052-1057.

Voudouris NJ, Peck CL, Coleman G (1990) The role of conditioning and verbal expectancy in the placebo response. Pain 43:121-128.

Wager TD, Rilling JK, Smith EE, Sokolik A, Casey KL, Davidson RJ, Kosslyn SM, Rose RM, Cohen JD (2004) Placebo-induced changes in FMRI in the anticipation and experience of pain. Science 303:1162-1167.

Wager TD, Scott DJ, Zubieta JK (2007) Placebo effects on human \{micro\}opioid activity during pain. Proc Natl Acad Sci U S A 104:11056-11061.

Wagner U, Gais S, Born J (2001) Emotional memory formation is enhanced across sleep intervals with high amounts of rapid eye movement sleep. Learn Mem 8:112-119.

Wagner U, Gais S, Haider H, Verleger R, Born J (2004) Sleep inspires insight. Nature 427:352-355.

Walker MP, Stickgold R (2004) Sleep-dependent learning and memory consolidation. Neuron 44:121-133.

Walker MP, Liston C, Hobson JA, Stickgold R (2002) Cognitive flexibility across the sleep-wake cycle: REM-sleep enhancement of anagram problem solving. Brain Res Cogn Brain Res 14:317-324.

Wilson MA, McNaughton BL (1994) Reactivation of hippocampal ensemble memories during sleep. Science 265:676-679.

Zubieta JK, Bueller JA, Jackson LR, Scott DJ, Xu Y, Koeppe RA, Nichols TE, Stohler CS (2005) Placebo effects mediated by endogenous opioid activity on mu-opioid receptors. J Neurosci 25:7754-7762. 